\title{
Design of a 150-miniature detectors 3D core-mapping system for the CROCUS reactor
}

\author{
Fanny Vitullo ${ }^{1}$, Vincent Lamirand ${ }^{1,2}$, Klemen Ambrožič ${ }^{1}$, Laurent Braun ${ }^{1}$, Daniel Godat ${ }^{1}$, \\ Pavel Frajtag ${ }^{1}$, and Andreas Pautz ${ }^{1,2}$ \\ ${ }^{1}$ École polytechnique fédérale de Lausanne (EPFL), Switzerland \\ ${ }^{2}$ Paul Scherrer Institut (PSI), Switzerland \\ fanny.vitullo@epfl.ch, vincent.lamirand@epfl.ch
}

\begin{abstract}
The present article provides an overview of the design of a three-dimensional (3D) full-core mapping system for the CROCUS reactor, operated at the École polytechnique fédérale de Lausanne (EPFL), Switzerland. The system is composed of $\mathbf{1 4 9}$ miniature neutron detectors distributed within the core double lattice at three main axial levels. The miniature detector technology is based on the optimization of the well-proven coupling of a miniature ZnS: ${ }^{6} \mathrm{LiF}$ (Ag) scintillator to a state-of-the-art silicon photomultiplier (SiPM) via jacketed optical fibers. The challenges in the mechanical design, the detector optimization, the core criticality, and the development of the acquisition electronics are strongly interconnected and their combination is addressed in this article. The 3D full-core mapping system is foreseen to be installed in CROCUS in autumn 2021 and it will pave the way for the investigation of 3D dynamic phenomena in nuclear reactor cores.
\end{abstract}

Keywords - Reactor instrumentation, 3D core-mapping, miniature neutron detector, in-core neutron detection, CROCUS.

\section{INTRODUCTION}

$\mathrm{T}$ HE interest in studying space-dependent neutronics phenomena in nuclear reactor cores has grown in recent years among the scientific community. The knowledge of detailed three-dimensional (3D) power distributions, local flux perturbations due to dynamic events, and the spatialization of neutron noise induced by mechanical perturbations would allow to determine and optimize reactor operational safety margins in both normal and accidental conditions. As of today, the spatial and/or temporal behavior of a reactor core can be predicted with high-fidelity by advanced neutronics codes. However, the validation of their $3 \mathrm{D}$ high-fidelity capabilities is non-trivial due to the limited availability of extensive and localized in-core neutron flux measurements in reference reactor cores [1]. Indeed, the challenges in the installation of $3 \mathrm{D}$ detection systems to gather such data are numerous. First of all, positioning a great number of in-core neutron detectors requires good reactor accessibility and inter-pin spaces fitting neutron detectors of a suitable size. Secondly, both the selected detectors and their acquisition chain must be easily produced in series at an acceptable cost.

In this framework, the zero-power research reactor CROCUS, operated at the École polytechnique fédérale de Lausanne (EPFL) was selected as a perfect candidate for the installation of a 3D full-core mapping system. A 3D measuring system in CROCUS will provide valuable insight on the propagation of neutron modulation in the core, thanks to the presence of several mechanical perturbation experiments, including the COLIBRI fuel rod oscillator [2], [3], the PISTIL rotating absorber [4], and a linear oscillating absorber currently under development. In addition, CROCUS is a readily available reactor with low technological uncertainties [5], where it would be possible to accurately position $\sim 150$ neutron detectors within the whole core volume upon the design of a proper mechanical supporting structure. At the same time, the Laboratory for Reactor Physics and Systems Behaviour (LRS) at EPFL in collaboration with the Paul Scherrer Institut (PSI) had developed a prototype of a miniature detector with an active volume below $1 \mathrm{~mm}^{3}$ [6], [7]. The detection technology is based on the well-proven coupling of a miniature $\mathrm{ZnS}:{ }^{6} \mathrm{LiF}(\mathrm{Ag})$ scintillator to a state-of-the-art silicon photomultiplier (SiPM) via jacketed optical fibers. After optimization in dimensions, processing electronics, and acquisition scheme, such detectors suit the needs in terms of size and scalability for the setup of a 3D mapping system in CROCUS.

In this article, the full design of the 3D core-mapping system for the CROCUS reactor is presented. The synergies between neutron detectors physics and size, mechanical design, core criticality, and electronics acquisition are highlighted. In the first section, the custom mechanical design of the system is described. Secondly, the optimization of the miniature neutron detector prototype is discussed, followed by a study on the reactivity impact of the whole system. Finally, the digital acquisition electronics, developed in-house at LRS and tailored for the 3D core-mapping system, is presented.

\section{MECHANICAL DESIGN}

\section{A. The CROCUS reactor}

CROCUS is a zero-power research reactor operated at EPFL and dedicated to education and research [8]. The cylindrical reactor core is composed of low-enriched and aluminumcladding fuel rods inserted into an open pool containing demineralized water acting as moderator and reflector. In the current configuration, the core active zone is divided into two different fuel zones with distinct lattice dimensions. The inner zone is composed of 336 uranium dioxide $\left(\mathrm{UO}_{2}\right)$ rods arranged in a squared lattice with a pitch of $18.37 \mathrm{~mm}$. In the surrounding outer zone, 176 metallic uranium rods $\left(\mathrm{U}_{\mathrm{met}}\right)$ are organized in a 
square lattice as well, but with a $29.17-\mathrm{mm}$ pitch. In the west part of the outer fuel zone, a fuel rod oscillator device was installed as part of the COLIBRI experimental program [2]. The device allows to oscillate up to $18 \mathrm{U}_{\text {met }}$ fuel rods (called "COLIBRI rods" in this article) of up to $\pm 2.5 \mathrm{~mm}$ around nominal with a frequency in the $\mathrm{Hz}$ range. All the rods in the core are kept in a vertical position by two octagonal aluminum grids positioned at $1 \mathrm{~m}$ from each other. A 1-mm cadmium layer is included in both grids to limit the axial neutron leakages.

The maximum allowed fission power is limited to $100 \mathrm{~W}$, corresponding to a neutron flux of about $2.5 \times 10^{9} \mathrm{~cm}^{-2} \cdot \mathrm{s}^{-1}$ at the core center. The core criticality can be controlled in two different ways: by adjusting the water level using a spillway, or by the insertion and withdrawal of two $\mathrm{B}_{4} \mathrm{C}$ absorber control rods in two aluminum tubes, which can also be used as experimental channels. Six independent safety systems are installed in the core: two cruciform cadmium safety blades falling into the $\mathrm{UO}_{2}$ lattice and four expansion valves for quickly emptying the water tank. Each of the six systems is enough to shut down the reactor. A 3D model of CROCUS is shown in Fig.1.

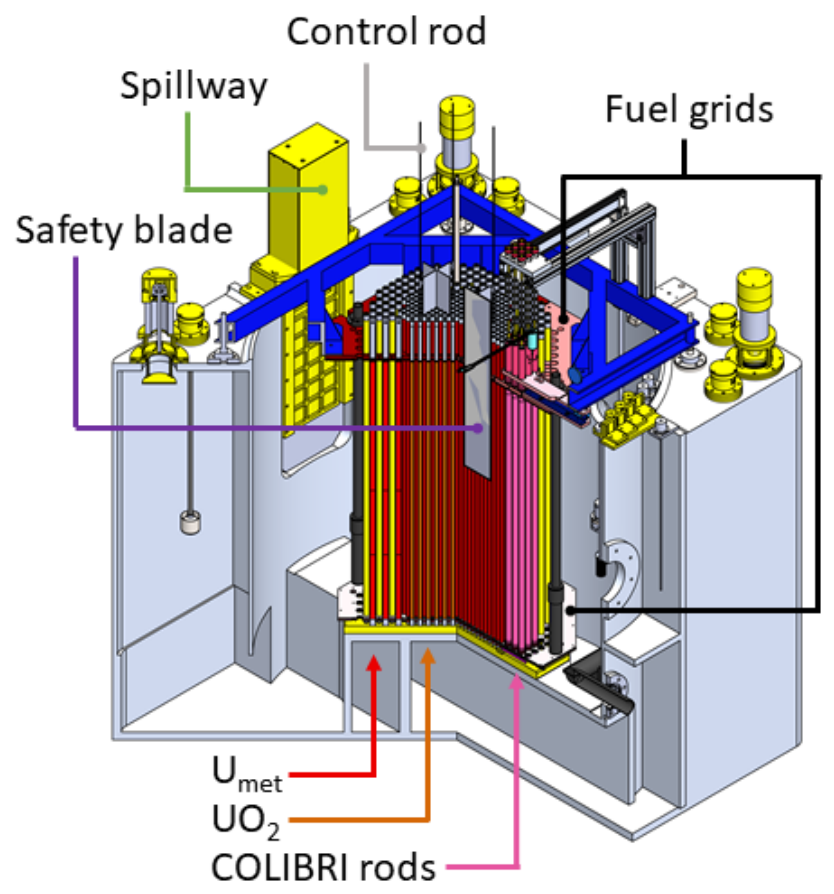

Fig. 1. 3D section of the CROCUS reactor model equipped with the COLIBR fuel rod oscillator.

\section{B. Conceptual design and boundary conditions}

The idea behind the installation of a 3D full-core mapping system in CROCUS consists in the positioning of $\sim 150$ thermal neutron detectors within the fuel lattices, split among three identical axial layers and arranged to have the best possible mapping of induced perturbations in the core, e.g. fuel rod oscillations within the COLIBRI program, absorbers insertions or rotations, etc. As a consequence, an asymmetry in the detector distribution can be observed in the horizontal cut shown in Fig. 2, where a greater number of detectors is envisaged to be positioned within the $18 \mathrm{U}_{\text {met }}$ oscillating rods. In the same optic, the addition of two more layers of detectors in the proximity of the control rods guide tubes (and on the symmetrical rods) would allow for a study of the localized perturbation induced by a control rod extraction. In conclusion, each of the three axial main layers will hold 47 detectors, plus two extra layers of 4 detectors each, for a total of 149 detectors in the CROCUS core.

However, installing 149 neutron detectors within the CROCUS core is a non-trivial goal. These detectors require a mechanical structure that holds and keeps them in the selected positions without having to modify the basic design of the reactor described in the previous section. The mechanical system must be designed to be as practical as possible for its out-of-vessel loading, of easy manufacturing, and respectful of the CROCUS safety standards. Indeed, there must be no contact or friction between the detectors or the structure and the aluminum cladding of the fuel rods. All the additional components must be chemically inert in demineralized water and they have to minimize the reactivity impact on the core criticality (see Section IV for a detailed reactivity study).

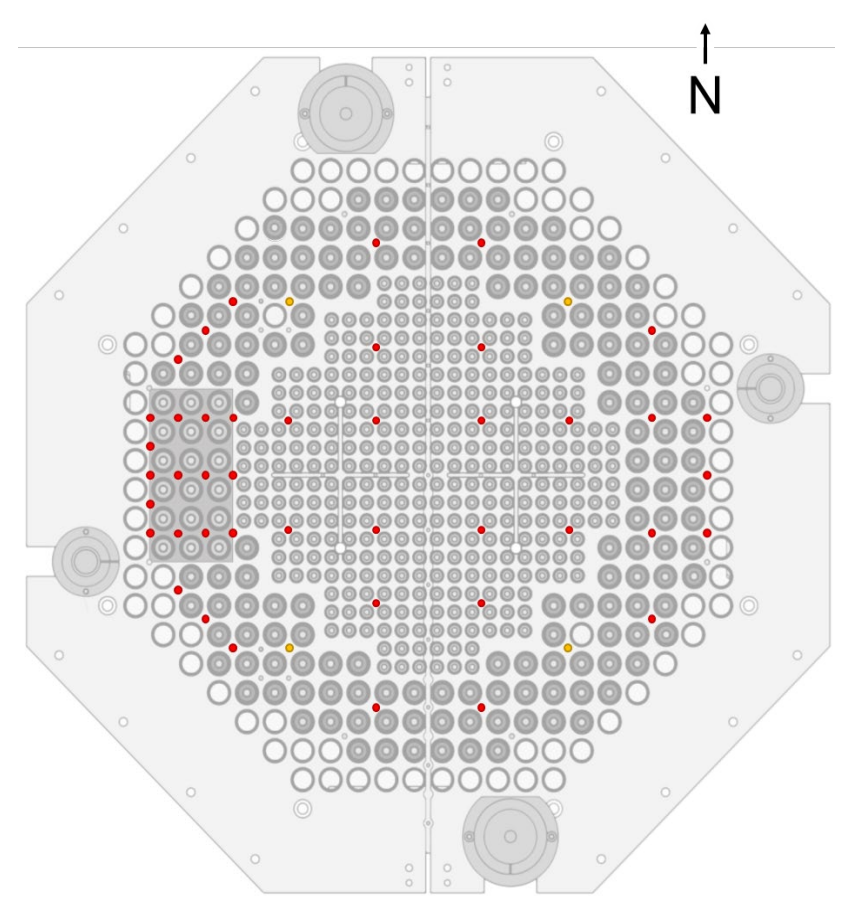

Fig. 2. Horizontal cut of CROCUS representing one of the three main layers of the $3 \mathrm{D}$ mapping system. Red dots represent the position of interest to install neutron detectors, while in yellow are indicated the positions where two supplementary intermediate layers of detectors are added.

\section{Mechanical constraints}

In the CROCUS reactor, a vertical insertion of the miniature detectors in the inter-pin space is made impractical by the presence of the upper fuel grid. Opening a passage for the optical fibers would require delicate machining of the grid to fit the purpose. Therefore, a horizontal insertion was preferred for the 3D mapping system, even though the peculiar double-lattice arrangement of CROCUS introduces limitations. Figure 3 shows the positions at which it is possible to cross both lattices and indicates the maximum available space in $\mathrm{mm}$ to perform full or partial straight crossings. Partial crossings arise from the presence of the cruciform safety blades in the core center, whose run must be kept clear at all times for safety reasons. However, these partial crossings can be performed from both north and south directions. Looking at Fig. 3, and excluding the 
partial crossing of $3.48 \mathrm{~mm}$, the minimum space available between fuel rods is equal to $4.59 \mathrm{~mm}$. Hence, the maximum allowed thickness of material cutting across the core was set to $4 \mathrm{~mm}$ to leave a margin for possible mechanical tolerances. The 4-mm limit remains valid also when the oscillating fuel rod device is displacing the 18 fuel rods indicated in pink in Fig. 3 by $\pm 2.5 \mathrm{~mm}$ and reducing the $9.87 \mathrm{~mm}$ margin to $7.37 \mathrm{~mm}$.
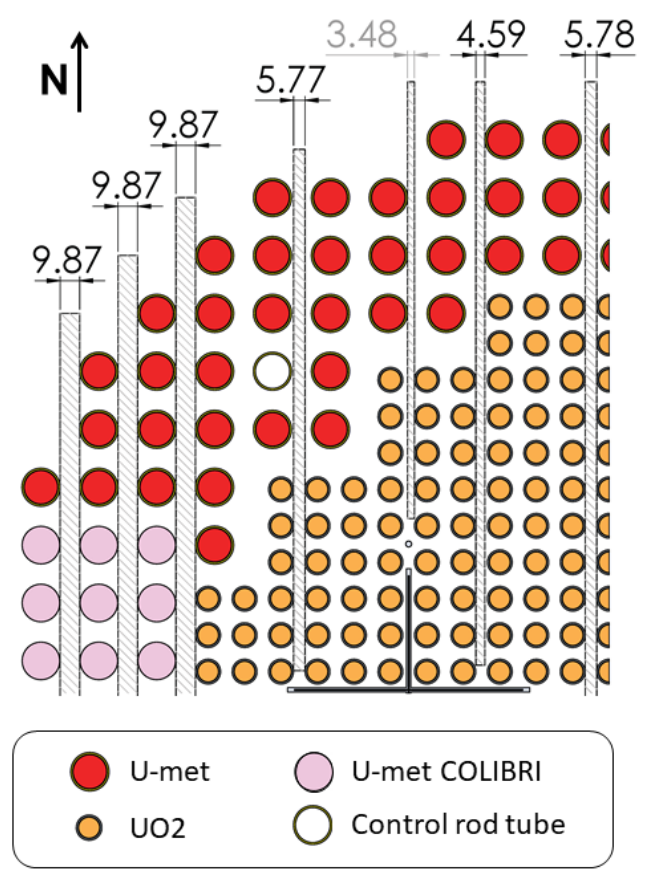

Fig. 3. North-west portion of the horizontal section of the CROCUS reactor with indication of the locations available to cross in a straight line the double fuel lattice. The maximum space available for the crossing is indicated in $\mathrm{mm}$.

\section{Final design of supporting structure}

Based on the lateral insertion strategy of the 149 detectors and the 4-mm space limit, a tailored mechanical structure to load and hold the 3D full-core mapping system has been designed in-house at LRS. The SolidWorks model of the system installed in the CROCUS core is shown in Fig.4. The main elements composing the $3 \mathrm{D}$ full-core mapping system are:

- 50 rectangular "rulers" of polyoxymethylene (POM-C) with a thickness of $4 \mathrm{~mm}$ guiding and positioning the neutron detectors at the intended position. Black POM-C was selected because of its good resistance in water, its good light opacity and its density comparable to that of water $\left(1.41 \mathrm{~g} / \mathrm{cm}^{3}\right)$. Depending on the lattice arrangement, rulers can be crossing the whole core or being split in two to accommodate the presence of the cruciform safety blades;

- 52 horizontal supporting rods made of anodized aluminum for holding and positioning the rulers among fuel rods at 5 different axial layers. The three main levels (L1, L2 and L3 in Fig. 4) with 47 detectors each are positioned in a way that the detector's active part is located at $15,50,85 \mathrm{~cm}$ of core height respectively. The two additional layers close to control rods guide tubes (LM1 and LM2 in Fig.4) are at 30 and $70 \mathrm{~cm}$;

- 8 vertical supporting rods of anodized aluminum in ex-core positions to support the horizontal rods at the different axial levels;

- 8 vertical supporting rods in anodized aluminum in in-core position to hold the not-crossing rulers. Dedicated adaptation pieces in aluminum and POM-C allow to attach these rods to the upper and lower fuel grid of CROCUS.

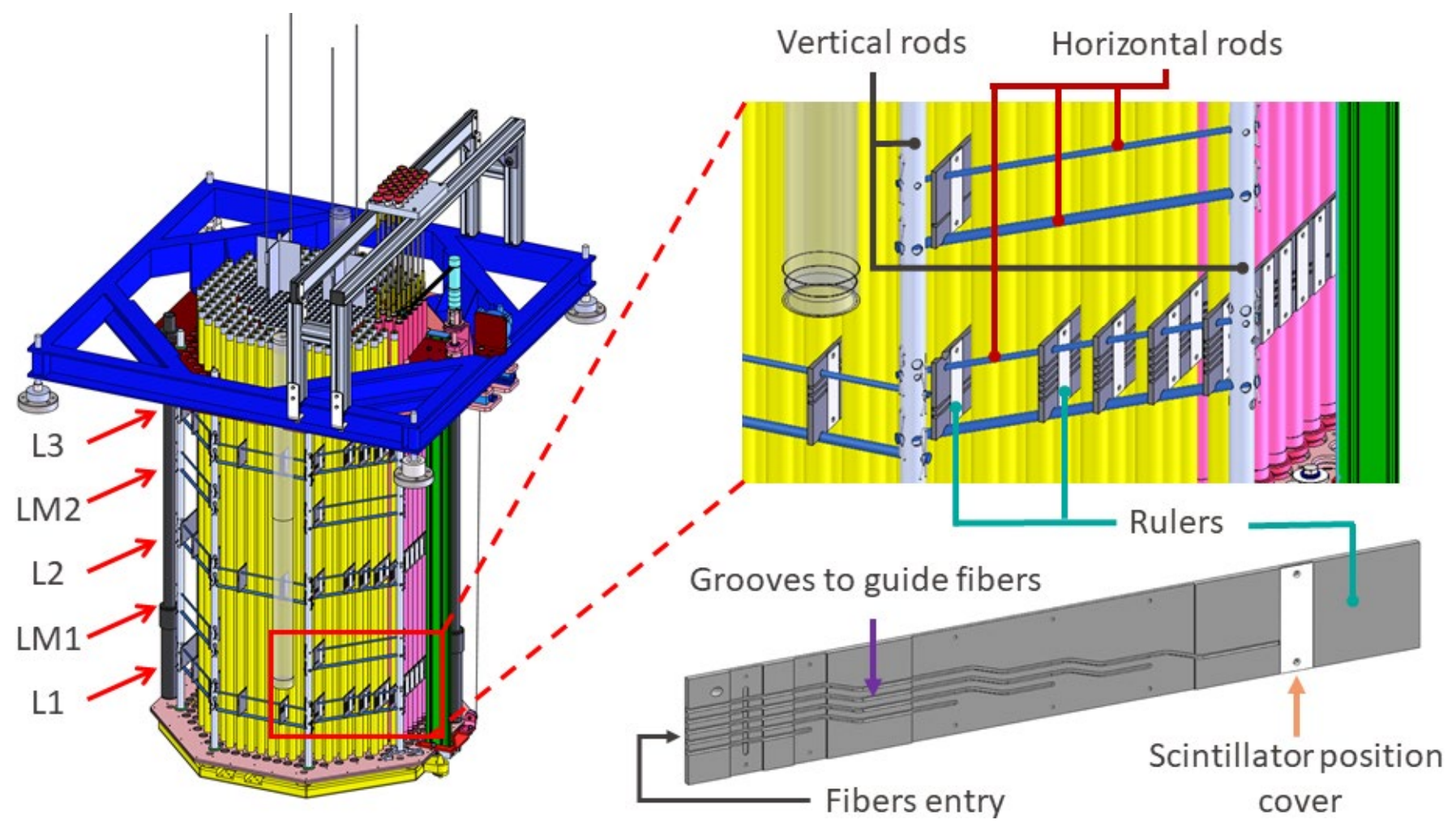

Fig. 4. SolidWorks model of the 3D full-core mapping system installed in the CROCUS reactor core and zoom on the main components of the system. 
Aside from the standard components mentioned above, small variations are necessary to accommodate special design needs arising from the presence of numerous experiments and customizations in CROCUS. An example is represented by the vertical rod placed at the north side of the COLIBI rods, which cannot be inserted as the other 7 due to the presence of the fuel rod oscillator, and thus has two special grid attachments.

Mock-up pieces of both rulers and supporting structure have been produced to assess the mechanical stability of the assembly. Pictures of a test ruler and the mock-up mechanical structure are reported in Fig. 5.

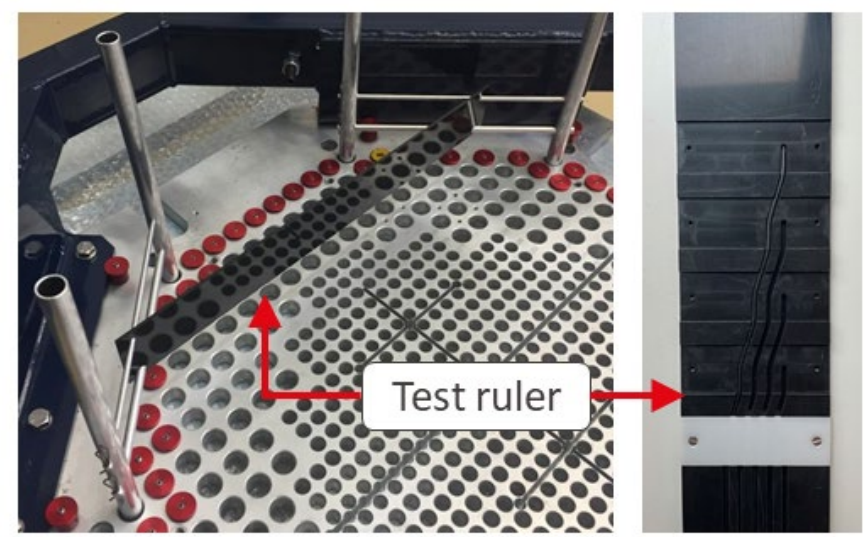

Fig. 5. Mock-up mechanical structure to support a test ruler.

\section{OPTIMIZATION OF DETECTOR DESIGN}

\section{A. First prototype design}

The first prototype of a miniature neutron detector was developed and successfully tested at EPFL in collaboration with PSI in 2018 [6], [7]. This first development version was composed by a $1 \mathrm{~mm}^{2} \times 0.2 \mathrm{~mm} \mathrm{ZnS}:{ }^{6} \mathrm{LiF}(\mathrm{Ag})$ screen [9] positioned at the tip of a $2-\mathrm{mm}$ core optical fiber [10]. The blue light produced (with peak emission at $450 \mathrm{~nm}$ [9]) after a thermal neutron interaction with ${ }^{6} \mathrm{Li}$ in the screen is transported through the optical fiber to a silicon photomultiplier (SiPM) [11], where each photon event is translated into an electrical signal after proper processing. Neutron events are then discriminated from background on the basis of the photondensity variation in the stream of photon pulses. Indeed, assuming good dark conditions of the system, only few sparse pulses correspond to the background, while a step-wise and longlasting (few tens of $\mu$ s [9]) increase of the photon density is visible after a neutron interaction. In this first development version, the discrimination and the thermal neutron counting were performed using an analog processing electronics (see Section V for more details on processing electronics). Figure 6 summarizes the detection principle.

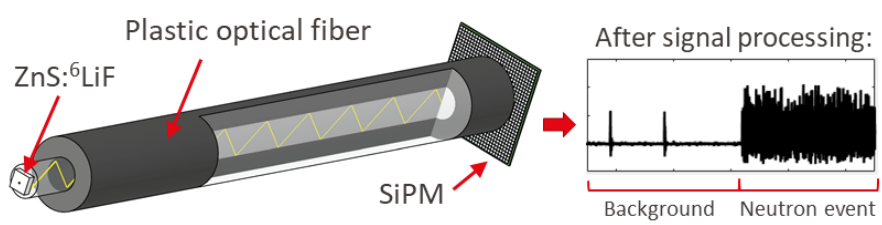

Fig. 6. Schematic of a miniature neutron detector working principle.
The first testing and characterization of the prototype showed that a detector of such reduced dimensions $\left(<1 \mathrm{~mm}^{3}\right)$ maintains a good sensitivity in terms of neutron counting in CROCUS, up to a total neutron flux of $\sim 10^{7} \mathrm{n} / \mathrm{cm}^{2} / \mathrm{s}$ [6], [7]. It was proven that the detector is insensitive to gammas due to its small active volume and discrimination scheme [7], and the perturbation induced in the flux map is minimal [12], [13]. All these features, combined with the detector low-cost and easy-manufacturing, make the technology the perfect choice for a distributed coremapping system, as long as it is possible to acquire data simultaneously from a large number of detectors in a costeffective way (see Section V).

\section{B. Optical fiber optimization}

Although the excellent performance of the first prototype version proven in [7], [12], and [13], the outer fiber diameter of $3 \mathrm{~mm}$ represents a significant constraint when the miniature detectors are envisaged to be inserted in the CROCUS reactor core via the 4-mm thick POM-C rulers. A 3-mm fiber would leave a minimal plastic thickness that might compromise the mechanical stability of the rulers. Therefore, four different plastic optical fibers were tested and compared to the prototype version (called fiberA) to find a tradeoff between diameter and performances. The four fibers, whose characteristics are listed in Tab. 1, are all produced by the ESKA Mitsubishi Chemical Company [14].

TABLE I

OPTICAL FIBERS TESTED FOR OPTIMIZATION

\begin{tabular}{lllll}
\hline Name & \multicolumn{1}{c}{ Model } & $\begin{array}{c}\text { Outer } \\
\text { diameter } \\
(\mathrm{mm})\end{array}$ & $\begin{array}{c}\text { Core } \\
\text { diameter } \\
(\mathrm{mm})\end{array}$ & $\begin{array}{c}\text { Jacket } \\
\text { thickness } \\
(\mathrm{mm})\end{array}$ \\
\hline fiberA & SH8001 & 3 & 2 & 0.5 \\
fiberB & SH4001 & 2.2 & 1 & 0.6 \\
fiberC & SH4001-1.3 & 1.3 & 1 & 0.15 \\
fiberD & SH2001 & 1 & 0.5 & 0.25 \\
\hline
\end{tabular}

The four fibers were tested in blank conditions, meaning without counting neutrons, in order to assess their background level independently from the measurement conditions. Signals corresponding to background photons arise from the lighttightening quality of the fiber tip coverage, the fiber jacketing, and the coupling with the SiPM. Considering that all four optical fibers had the same front-end tip cover and an identical pairing with the SiPM, the number of background photons filtering from the polyethylene jacket in $1 \mathrm{~ms}$ was measured. The counting was performed using a CAEN V2495 FPGA [15] loaded with the PLUscaler firmware [16]. The test was repeated in two different light conditions to assess whether laboratory environmental conditions could bias the measurements.

The results are presented in Fig. 7 that clearly shows how the jacket thickness is a key parameter in the selection of the optical fiber. In dark conditions, the number of background photons is of the same order of magnitude for the four optical fibers. However, in daylight conditions the number of background photons increases significantly for optical fibers with a thin jacket with respect to their counting in the dark. The number 


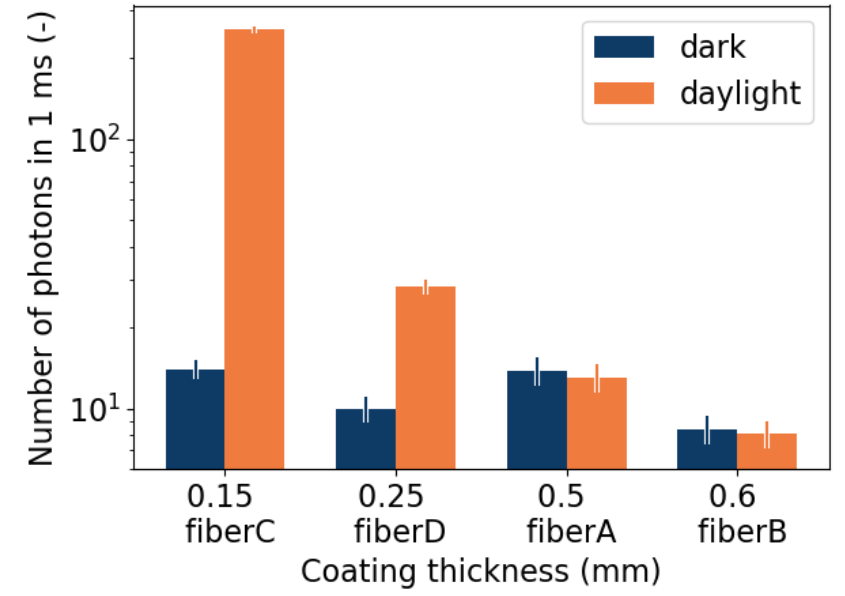

Fig. 7. Number of background light photons filtering from the optical fiber jacket in $1 \mathrm{~ms}$ as a function of the jacket thickness, in two different light conditions.

of photons measured in daylight decreases with the increase of the jacket thickness, up to a point where there is no difference between daylight or darkness conditions.

Therefore, above a jacket thickness of $0.5 \mathrm{~mm}$, the optical fiber is properly light-shielded and unaffected by the external light conditions. Looking at Fig. 7, the only two optical fibers having a jacket thickness above $0.5 \mathrm{~mm}$ are fiberA (SH8001), corresponding to the prototype fiber in [7], and fiberB (SH4001), having a polymethyl methacrylate (PMMA) core of $1 \mathrm{~mm}$ in diameter and an outer diameter of $2.2 \mathrm{~mm}$. According to the requirements, fiberB represents the perfect combinations between the optical fiber performances and the dimensional constraints set by the design of the $3 \mathrm{D}$ full-core mapping system.

\section{Final detector design for $3 D$ core-mapping system}

The choice of fiberB, having a core diameter of $1 \mathrm{~mm}$, lead to the optimization of the $\mathrm{ZnS}:{ }^{6} \mathrm{LiF}(\mathrm{Ag})$ screen so that the level of thermal neutron sensitivity is maximized. A new Scintacor ND2:1 foil with $0.225 \mathrm{~mm}$ thickness [9] was precisely cut at EPFL into square pieces of $0.66 \mathrm{~mm} \times 0.66 \mathrm{~mm}$ using a wire-cutting technique. This screen size allows for a good coverage of the fiber tip and a possible reduction of the reactivity impact if compared to the prototype version (see next Section).

The $0.66 \times 0.66 \mathrm{~mm}$ screen is glued at the sanded and uncovered tip of the optical fiber PMMA core. An epoxidic adhesive called Araldite [17] is used due to its excellent resistance in water, fast setting and good transparence. With this method, the $\mathrm{ZnS}:{ }^{6} \mathrm{LiF}(\mathrm{Ag})$ screen is embedded in the glue itself and both unwanted movements or loss of the screen in the CROCUS core are prevented.

Figure 8 shows the final optimized design chosen to build the 149 miniature neutron detectors for the 3D full-core mapping system in CROCUS. Although the good accuracy in the $\mathrm{ZnS}:{ }^{6} \mathrm{LiF}(\mathrm{Ag})$ screens cutting and gluing, a detector intercalibration with a $\mathrm{Pu}-\mathrm{Be}$ source available at LRS is envisaged to be carried out before the system installation in the CROCUS core.

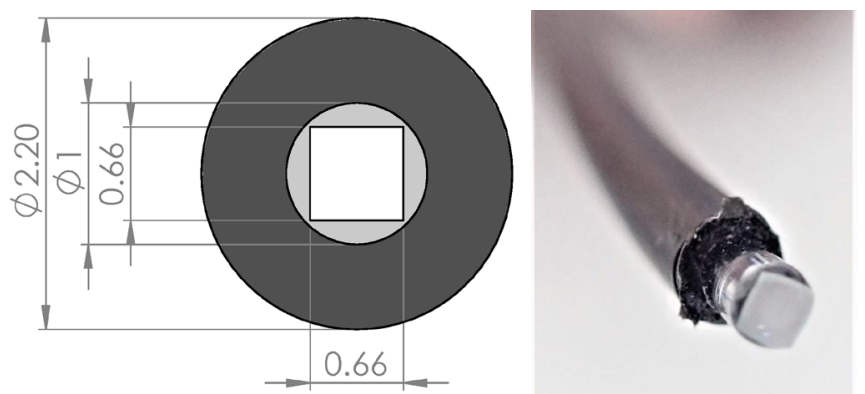

Fig. 8. Horizontal section in scale (a) and picture (b) of the SH4001 optical fiber with a squared $0.66 \times 0.66 \times 0.225 \mathrm{~mm} \mathrm{ND} 2: 1$ screen glued at the tip.

\section{REACTIVITY IMPACT}

The 3D core-mapping system designed for the CROCUS reactor, composed by 149 optimized miniature neutron detectors and the mechanical structure, will have an impact on the reactor operations in terms of criticality. It is foreseen that the $149 \mathrm{ZnS}:{ }^{6} \mathrm{LiF}(\mathrm{Ag})$ scintillator screens will introduce a strong negative reactivity insertion, which could be partially counteracted by the positive reactivity effect induced by the installation of the POM-C rulers having a slightly higher density than water. By understanding the balance between these concurrent effects it is possible to assess if the reactor can be operated within the authorized core configurations and in the respect of the safety limits. Hence, the core criticality is studied computationally via Monte Carlo simulations performed with the MCNPX-2.4 code [18], as required by the Swiss safety authority. A detailed MCNPX model of a typical configuration of CROCUS was used as a baseline and reference.

A key parameter to obtain reliable simulation results is represented by the material composition of the $\mathrm{ZnS}:{ }^{6} \mathrm{LiF}(\mathrm{Ag})$ screens, and in particular by the level of enrichment of ${ }^{6} \mathrm{Li}$. Based on the supplier data and in order to obtain conservative results, a $95 \%{ }^{6} \mathrm{Li}$ enrichment was used, and the resulting reactivity worth of the screen was compared with experimental results. Two miniature neutron detectors with different sensitive volume were inserted alternatively in the north-west control rod guide tube of CROCUS and the critical level variation with respect to the standard configuration was recorded. The results of both experiments and simulations are reported in Table II, where a good agreement in the reactivity worth range of few negative pcm is shown.

TABLE II

MEASURED AND SIMULATED RESULTS FOR THE REACTIVITY WORTH IN CROCUS OF TWO MINIATURE NEUTRON DETECTORS WITH DIFFERENT SENSITIVE VOLUME [21]. SIMULATIONS WERE PERFORMED WITH JEFF 3.1 [19].

\begin{tabular}{lll}
$\begin{array}{c}\text { Screen volume } \\
\left(\mathrm{mm}^{3}\right)\end{array}$ & \multicolumn{1}{c}{$\begin{array}{c}\text { Measured reactivity worth } \\
(\mathrm{pcm})\end{array}$} & $\begin{array}{c}\text { Simulated reactivity worth } \\
(\mathrm{pcm})\end{array}$ \\
\hline 0.2 & $-1.2 \pm 0.2$ & $-1 \pm 2$ \\
0.09 & $-0.7 \pm 0.2$ & $-1 \pm 1$ \\
\hline
\end{tabular}

Having confirmed the material composition, the entire 3D core-mapping system was implemented in the MCNPX model. For the sake of simplicity, rulers are modeled as single parallelepipeds, with no distinction between the optical fiber and the ruler material because both are made of similar plastics. The $0.66 \times 0.66 \times 0.225 \mathrm{~mm} \mathrm{ZnS}:{ }^{6} \mathrm{LiF}(\mathrm{Ag})$ screens are modeled 
in the predetermined positions (see Fig. 2). The rest of the supporting structure was included in the model following as much as possible the previously described mechanical design. Two different nuclear data libraries were used for the simulations: JEFF 3.1 [19] and ENDF/B-VII.1 [20]. The simulation results are presented in Table III.

TABLE III

SIMULATED REACTIVITY WORTH FOR THE 3D CORE-MAPPING SYSTEM [21].

\begin{tabular}{lcccc}
\hline Configuration & $\begin{array}{c}\text { JEFF-3.1 } \\
\mathrm{K}_{\mathrm{eff}}\end{array}$ & $\begin{array}{c}\text { JEFF-3.1 } \\
\text { worth } \\
(\mathrm{pcm})\end{array}$ & $\begin{array}{c}\text { ENDF/B-VII.1 } \\
\mathrm{K}_{\mathrm{eff}}\end{array}$ & $\begin{array}{c}\text { B-VII.1 } \\
\text { worth } \\
(\mathrm{pcm})\end{array}$ \\
\hline $\begin{array}{l}\text { Standard } \\
\begin{array}{l}\text { With 3D } \\
\text { core-mapping }\end{array}\end{array}$ & $1.00240 \pm 1 \mathrm{pcm}$ & 0 & $1.00289 \pm 2 \mathrm{pcm}$ & 0 \\
\hline
\end{tabular}

The results in Table III show that the general effect of mounting the 3D full-core mapping system in CROCUS is a negative reactivity insertion. The strongest negative reactivity insertion corresponds to $-51 \pm 3 \mathrm{pcm}$, which is well below the maximum available reactivity reserve of CROCUS of $+200 \mathrm{pcm}$. Therefore, the CROCUS reactor with the 149-detectors system installed in its core can be safely operated within its standard limits. At the same time, a request for the addition of two $U_{\text {met }}$ rods in the core periphery has been submitted to the Swiss safety authority to avoid that the future installation of additional absorbing devices would deplete the core's reactivity reserve.

\section{DIGITAL ACQUISITION CHAIN}

The first prototype model of miniature neutron detector described in Section III.A was using a processing electronics developed at PSI to transform SiPM currents into a stream of photon events, and an analog read-out electronics assembled at LRS to count and discriminate thermal neutron events from the background. Despite the good performance and excellent reliability of this electronic system (more details can be found in [7]), the latter presents some limitations whether to be employed for an extensive core-mapping system. In particular, the acquisition part was composed by standard and expensive electronic modules that are limiting the acquisition to only a few channels simultaneously. This restriction on the system parallelization at a reasonable price prompted the development of a new electronics.

In a first attempt of upgrading the system, a novel processing electronic board was developed internally at LRS to improve photon collection efficiency, pulse shortening, as well as light noise reduction. Three boards, composed by a SiPM and a fast pre-amplifier stage, were built and tested in [13]. Their good performances paved the way for the development of an extensive electronics tailored for the $3 \mathrm{D}$ core-mapping.

\section{A. Novel 32-channels processing electronics}

Six novel stand-alone electronic modules based on the three prototype boards were developed and built in-house at LRS. Each module is able to process simultaneously the incoming signal from 32 different miniature neutron detectors. The use of five modules together covers 160 channels and thus the needs of the $3 \mathrm{D}$ core-mapping system. The sixth module is a backup.

The module front-end, visible in Fig. 9(a), presents 32 openings where each optical fiber can be connected and coupled with its respective SiPM via a black plastic cylinder. The cylinders are separating and keeping in the dark the 32 SiPMs to minimize background noise and cross-talk events. Although the cylinders and the external structure are designed to minimize the light filtering, additional layers (a wardrobe and a black cloth) are foreseen to be added to maximize the coverage. Each SiPM output is sent to one of 32 separated processing boards produced following the prototype design and shown in Fig. 9(c). The boards are all mounted on a motherboard visible in Fig. 9(b) from above. Each board is dedicated to the conversion of the corresponding SiPM current signal into a photon counting stream through a user-selected threshold level (called "photon threshold") for electronic noise removal. Both the $32 \mathrm{SiPMs}$ and the 32 processing boards are powered by an internal alimentation module. The bias voltage applied to the SiPMs and the photon threshold can be selected with knobs on the module front-end and their level can be visualized on a display.

The output of a single 32-channels module consists in the simultaneous photon counting stream in every of the 32 channels. These signals, using the LVDS standard, can be then sent to a dedicated FPGA module to discriminate neutron events and perform the neutron counting.
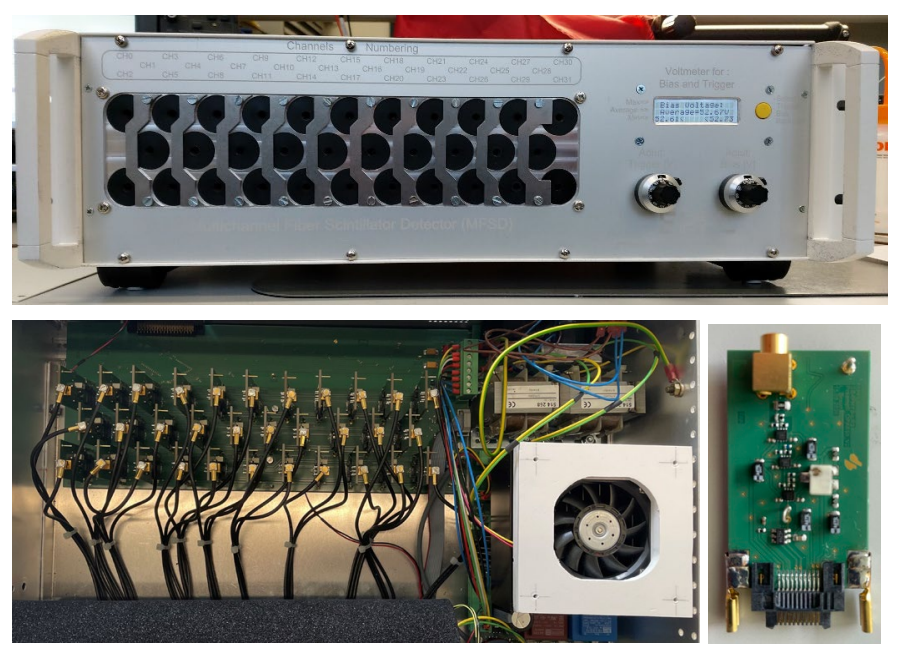

Fig. 9. Front-end (a) and internal (a) of a 32-channel module developed at LRS. One of the 32 fast-preamplifier board installed in the module (c).

\section{B. FPGA acquisition}

The 160 photon counting signal produced by the five 32 channels boxes can be treated at the same time by using an FPGA board. In this work, the general-purpose programmable CAEN V2495 [15] was used. The board has 64 build-in inputs that can be expanded to 160 adding three mezzanines boards in the expansion slots, accommodating the 160 photon counting signals. The board can be controlled and programmed through the USB interface, or as in this work via the V1718 VME bridge module [22].

A custom-made proprietary firmware to process photon counting into neutron counting was developed at PSI and in the present work is loaded into the V2495 User FPGA. The 
software counts the number of photons in fixed intervals of time, whose length can be user-selected and ranges from $5 \mathrm{~ns}$ to $1.125 \mu \mathrm{s}$. A moving-average based algorithm is then applied to the number of photons counted in each interval: the sum of photons in five consecutive intervals is compared with the sum of photons counted in the previous five. This moving-average technique allows to identify density variations in the number of photons and thus to recognize the dense and long stream of photons originating from a neutron event in the $\mathrm{ZnS}:{ }^{6} \mathrm{LiF}(\mathrm{Ag})$ screen. To perform this discrimination, a limit called "digital threshold" is set by the user to distinguish between low photondensity occurrences, i.e. background, and the high light-density events corresponding to neutrons. An additional parameter called "blocking window" can be tweaked by the user to avoid erroneous discriminations.

In conclusion, thermal neutron events can be counted and recorded according to the selected digital threshold and blocking window. The entire acquisition scheme is performed simultaneously by the FPGA in all the 160 channels available. Hence, the signals coming from the 149 miniature neutron detectors composing the 3D full-core mapping system can be acquired with a single affordable module.

\section{CONCLUSIONS}

A 3D full-core mapping system was designed for the CROCUS reactor. The system features the installation of 149 optimized miniature neutron detectors distributed within the core double lattice at three main axial levels with the support of a mechanical structure. The system was mechanically designed taking into account the safety limits, the dimensional constraints of the core, and the need for mapping the propagation of mechanical perturbations in the core. The final design is composed of 50 4-mm thick plastic rulers guiding and positioning the detectors at the desired positions. The rulers are kept in position by an aluminum structure surrounding the core.

The miniature neutron detector design was optimized to accommodate them in the 4-mm rulers. An optical fiber with an external diameter of $2.2 \mathrm{~mm}$ and a $1-\mathrm{mm}$ diameter core was chosen due to the excellent trade-off between dimension reduction and enhanced performances offered. The size of the detector sensitive part, the $\mathrm{ZnS}:{ }^{6} \mathrm{LiF}(\mathrm{Ag})$ screen, was adapted to the updated optical fiber by cutting it into squares of $0.66 \times 0.66 \times 0.225 \mathrm{~mm}$.

The reactivity impact of the entire 3D core-mapping system was assessed with MCNPX simulations. It was calculated that the entire assembly introduces a negative reactivity insertion of $-51 \pm 3 \mathrm{pcm}$, which allows to safely operate the reactor without exceeding its reactivity reserve.

Finally, the electronics detection chain was upgraded by developing six 32-channels processing modules and a dedicated FPGA acquisition scheme. The combination of the two allows for the simultaneous read-out, amplification, processing, and acquisition of up to 160 channels, corresponding to 160 miniature neutron detectors.

The installation of the full-core mapping system for the CROCUS reactor is planned for autumn 2021. After a proper detector inter-calibration, the system will mark a milestone in proving the feasibility of such a first-of-a-kind system and in providing valuable localized experimental data for the validation of high-fidelity neutronics codes.

\section{ACKNOWLEDGMENT}

The research leading to these results has received funding from the Euratom research and training programme 2014-2018 under grant agreement No 754316.

The authors would like to thank the IVICT Europe $\mathrm{GmbH}$ company for their availability and kindness in providing optical fiber samples.

\section{REFERENCES}

[1] Z. I. Böröczki, G. Klujber, G. Tolnai, B. Molnár, D. Légrády, F. Gabrielli, A. Rineiski, and M. Szieberth, "Simulation of a research reactor reactivity transient with deterministic and GPU-assisted Monte Carlo reactor kinetics codes," Eur. Phys. J. Plus 135, 281 (2020). Available: https://doi.org/10.1140/epjp/s13360-020-00280-4.

[2] V. Lamirand, P. Frajtag, D. Godat, O. Pakari, A. Laureau, A. Rais, M. Hursin, G. Perret, C. Fiorina and A. Pautz, "The COLIBRI experimental program in the CROCUS reactor: characterization of the fuel rods oscillator", EPJ Web Conf., 225 (2020) 04020. Available: https://doi.org/10.1051/epjconf/202022504020.

[3] V. Lamirand, A. Rais, S. Hübner, C. Lange, J. Pohlus, U. Paquee, C. Pohl, O. Pakari, P. Frajtag, D. Godat, M. Hursin, A. Laureau, G. Perret, C. Fiorina and A. Pautz, "Neutron noise experiments in the AKR-2 and CROCUS reactors for the European project CORTEX," EPJ Web Conf., 225 (2020) 04023. Available: https://doi.org/10.1051/epjconf/202022504023.

[4] Y. Jiang, B. Geslot, V. Lamirand, P. Leconte, "PISTIL, a reactivity modulation device to probe the transfer function of the research nuclear reactor CROCUS", presented at the ANIMMA2021 conference, Prague, Czech Republic, Jun. 21-25, 2021.

[5] U. Kasemeyer, R. Früh, J. M. Paratte, and R. Chawla, "International Reactor Physics Experiments Handbook (IRPhE)," no. 4440, OECD, Ed. 2007, p. 94.

[6] F. Vitullo, V. Lamirand, J.-B. Mosset, P. Frajtag, O. Pakari, G. Perret and A. Pautz, "Developing and testing a miniature fiber-coupled scintillator for in-core neutron counting in CROCUS," EPJ Web Conf., 225 (2020) 04018. Available: https://doi.org/10.1051/epjconf/202022504018.

[7] F. Vitullo, V. Lamirand, J.-B. Mosset, P. Frajtag, O. Pakari, G. Perret, and A. Pautz, "A mm 3 Fiber-Coupled Scintillator for In-Core Thermal Neutron Detection in CROCUS," IEEE Trans. Nucl. Sci., vol. 67, no. 4, pp. 625-635, Apr. 2020.

[8] V. Lamirand, "Installation nucléaire CROCUS - Rapport de sécurité," version 1.2, 2017.

[9] Scintacor, "Neutron screens," 2020. Available: https://scintacor.com/wpcontent/uploads/2015/09/Datasheet-Neutron-Screens-High-Res.pdf. Accessed on: August 2021.

[10] ESKA Mitsubishi Chemical Corporation, "Data sheet SH-8001," 2019. Available: https://i-fiberoptics.com/pdf/sh-8001.pdf. Accessed on: August 2021.

[11] Hamamatsu Photonics K.K., Solid State Division, "MPPC (Multi Pixel Photon Counter) - S13360 Series," in particular SH13360-3050PE, 2016. Available:

https://www.hamamatsu.com/resources/pdf/ssd/s13360 series kapd105 2e.pdf. Accessed on: August 2021.

[12] F. Vitullo, V. Lamirand, P. Frajtag, G. Perret and A. Pautz, "HIGHLY LOCALIZED AZIMUTHAL MEASUREMENTS IN THE CROCUS REACTOR TOWARDS THE VALIDATION OF HIGH-FIDELITY NEUTRONICS CODES," EPJ Web Conf., 247 (2021) 08014. Available: https://doi.org/10.1051/epjconf/202124708014.

[13] E. L. Brunetto, F. Vitullo, V. Lamirand, K. Ambrožič, D. Godat, M. Buck, G. Pohlner, J. Starflinger, A. Pautz, "High resolution measurements with miniature neutron scintillators in the SUR-100 zero power reactor," presented at the ANIMMA2021 conference, Prague, Czech Republic, Jun. 21-25, 2021.

[14] ESKA Mitsubishi Chemical Corporation. Cable products line-up. Available: https://www.pofeska.com/pofeskae/product/02/index.html. Accessed on: August 2021. 
[15] CAEN S.p.A., V2495 - Programmable Logic Unit PLUS. Available: https:/www.caen.it/products/v2495/. Accessed on: August 2021.

[16] CAEN S.p.A., FW2495SC - 160 Channels Latching Scaler for V2495 and DT5495. Available: https://www.caen.it/products/fw2495sc/. Accessed on: August 2021.

[17] Araldite. ARALDITE® RAPID 24ML. Available: https://www.goaraldite.com/products/epoxy-adhesives/araldite-rapid-24ml-syringe. Accessed on: August 2021.

[18] L. S. Waters, ed., MCNPX User's Manual, Version 2.4.0, Los Alamos National Laboratory report LA-CP-02-408 (Sept. 2002).

[19] OECD/NEA Data Bank, "The JEFF-3.1 Nuclear Data Library", JEFF Report 21, OECD/NEA Data Bank (2006).

[20] M.B. Chadwick, M. Herman, P. Obložinský, M.E. Dunn, Y. Danon, A.C. Kahler, D.L. Smith, B. Pritychenko, G. Arbanas, R. Arcilla, R. Brewer, D.A. Brown, R. Capote, A.D. Carlson, Y.S. Cho, H. Derrien, K. Guber, G.M. Hale, S. Hoblit, S. Holloway, T.D. Johnson, T. Kawano, B.C. Kiedrowski, H. Kim, S. Kunieda, N.M. Larson, L. Leal, J.P. Lestone, R.C. Little, E.A. McCutchan, R.E. MacFarlane, M. MacInnes, C.M. Mattoon, R.D. McKnight, S.F. Mughabghab, G.P.A. Nobre, G. Palmiotti, A. Palumbo, M.T. Pigni, V.G. Pronyaev, R.O. Sayer, A.A. Sonzogni, N.C. Summers, P. Talou, I.J. Thompson, A. Trkov, R.L. Vogt, S.C. van der Marck, A. Wallner, M.C. White, D. Wiarda, P.G. Young, "ENDF/BVII.1: Nuclear Data for Science and Technology: Cross Sections, Covariances, Fission Product Yields and Decay Data", Nucl. Data Sheets 112 (2011) 2887.

[21] V. Lamirand, F. Vitulo, K. Ambrožič, O. Pakari, L. Braun, D. GODAT, "Report on the development of fibre-based scintillator," CORTEX D2.3 report, 2021.

[22] CAEN S.p.A., V1718 - VME-USB2.0 Bridge. Available: http:/primisdb.caen.it/products/v1718/. Accessed on: August 2021. 